\title{
Article
}

\section{Approaches Toward Improving the Prognosis of Pediatric Patients With Glioma: Pursuing Mutant Drug Targets With Emerging Small Molecules}

Snape, Timothy J. and Warr, Tracy

Available at http://clok.uclan.ac.uk/12394/

Snape, Timothy J. ORCID: 0000-0003-2766-4491 and Warr, Tracy (2014)

Approaches Toward Improving the Prognosis of Pediatric Patients With Glioma: Pursuing Mutant Drug Targets With Emerging Small Molecules. Seminars in Pediatric Neurology, 22 (1). pp. 28-34.

It is advisable to refer to the publisher's version if you intend to cite from the work. http://dx.doi.org/10.1016/j.spen.2014.12.003

For more information about UCLan's research in this area go to http://www.uclan.ac.uk/researchgroups/ and search for <name of research Group>.

For information about Research generally at UCLan please go to http://www.uclan.ac.uk/research/

All outputs in CLoK are protected by Intellectual Property Rights law, including Copyright law. Copyright, IPR and Moral Rights for the works on this site are retained by the individual authors and/or other copyright owners. Terms and conditions for use of this material are defined in the policies page. 
Title: Approaches towards improving the prognosis of paediatric glioma patients: pursuing mutant drug targets with emerging small molecules

Authors: Timothy J. Snape ${ }^{\mathrm{a}}$ and Tracy Warr ${ }^{\mathrm{b}}$

Author Affiliations: ${ }^{a}$ School of Pharmacy and Biomedical Sciences, University of Central Lancashire, Maudland Building, Preston, Lancashire, PR1 2HE, UK

${ }^{\mathrm{b}}$ Brain Tumour Research Centre, University of Wolverhampton, Wulfruna Street, Wolverhampton, WV1 1LY, UK

\section{INTRODUCTION}

Gliomas represent approximately $70 \%$ of all paediatric brain tumours and the majority of these will be of astrocytic lineage. Low-grade astrocytoma (LGA) comprise predominantly pilocytic astrocytoma (WHO grade I) and, less commonly, diffuse astrocytoma (WHO grade II). Malignant or high-grade astrocytoma (HGA) include anaplastic astrocytoma and glioblastoma multiforme (WHO grades III and IV, respectively) and account for approximately $20 \%$ of paediatric astrocytoma. ${ }^{1}$ Treatment options for paediatric glioma patients are limited. Although LGA are relatively slow-growing tumours which can often be cured through surgical resection, a significant proportion of cases will recur. Both LGA and HGA tumours which are disseminated or located in deep-seated or functionally critical areas are inoperable, especially those in the brainstem, including diffuse intrinsic pontine glioma (DIPG). ${ }^{2}$ Adjuvant radiotherapy can contribute to significant neurological morbidity and conventional chemotherapy with alkylating agents has achieved limited improvement in progression free or overall survival. ${ }^{3-6}$ 
Paediatric astrocytomas differ considerably from their adult counterparts in the spectrum and frequency of their mutations. Many of the common genetic abnormalities in adult glioma are not present in the paediatric tumours, particularly amplification and/or overexpression of EGFR and VEGRF and deletion/mutation of PTEN and hence, small tyrosine kinase inhibitors targeting these receptors are ineffective for treating paediatric astrocytoma. Recently, high-throughput screening, particularly whole genome and transcriptome sequencing analyses, have identified new oncogenic mutations in BRAF, FGFR1, ACVR1 and PPDM1 and amplifcation and/or overexpression of MYB/MYBL1 and IGF1R in paediatric LGA and HGA, which could be exploited in novel therapeutic interventions.

For example, upregulation of the RAS-RAF-MEK-ERK pathway through activating mutations is frequent in all grade of paediatric astrocytoma. In LGA, the predominant molecular alteration (present in $>70 \%$ cases) is an oncogenic KIAA1549-BRAF fusion protein which lacks the N-terminus regulatory domain of BRAF whilst retaining the Cterminus BRAF kinase domain. ${ }^{7}$ Approximately 20\% HGA (and a subset of grade II astrocytoma), harbour the ${ }^{\mathrm{V} 600 \mathrm{E}}$ BRAF mutation, which is constitutively activated through a T to A mutation leading to a valine to glutamate substitution at residue $600 .{ }^{8}$ MAPK signalling may also be enhanced through gain of function mutations in FGFR1, predominantly in tumours arising in the midline or brain stem. Missense mutations or tandem duplications in the tyrosine kinase domain of FGFR1 result in constitutive activation of the receptor in a subset of approximately 7\% LGA and 12\% HGA. ${ }^{7,9,10}$

Furthermore, rearrangements truncating the C-terminal miRNA negative-regulatory domain and/or amplification of the transcription factors $M Y B$ or $M Y B L 1$ have recently been reported in $25 \%$ of cerebral grade II diffuse astrocytoma. ${ }^{7,11,12}$ Mutations in ACVR1, encoding a bone 
morphogenetic protein (BMP) type 1 receptor, have been identified in 32\% DIPG and 12\% mid-line HGA in conjunction with the histone H3.1pLys27Met mutation. ${ }^{10,}{ }^{13}$ Here, missense mutations are located in either the kinase domain or the inhibitory glycine-serine-rich domain and induce ligand-independent activation of the TGF- $\beta$ signalling pathway and increased SMAD phosphorylation. ${ }^{14,15}$

\section{DISCUSSION}

The key principle in this approach, that of enhancing the prognosis of paediatric glioma patients using small molecules, focusses on exploiting the key genetic differences (outlined above) between adult and paediatric tumours. It has been shown for a number of key cellular pathways and biomolecular targets that the genetic differences of these two patient groups is significant, which therefore presents cancer researchers with a unique approach to use them in development of therapies. For example, protein kinases have enjoyed much success in the treatment of cancer over the past couple of decades, but the three-dimensional structure of the kinase in its biologically relevant conformation, and indirectly its primary sequence, is of utmost importance for the ability of drugs to bind and inhibit the kinase ${ }^{16}$ Moreover, sensitivity to anticancer drugs is compromised when mutations in the target occur which cause the shape and functionality of the active site to change, since the drug no longer binds and elicits a response. This is the crux of the problem when trying to treat paediatric glioma with drugs designed for adult glioma. If the biological target in the paediatric cancer cell is no longer reminiscent of that for which the drug was originally designed (in the adult), it will not work as intended. Furthermore, unwanted side-effects may result instead. Therefore, through the rational design of new compounds using the mutated target as a template, the development of an arsenal of paediatric-selective drugs could be achieved, which are more efficient than existing treatments for paediatric tumours. 


\section{Aberrant expression and mutated targets}

Based on this set of tumour types, there appears to be two main pathways of relevance to enable the potentially selective-treatment of paediatric glioma: 1) the RAS-RAF-MEK-ERK pathway; 2) the PI3K/AKT/mTOR pathway. Whilst other targets have been outlined above, the principle behind our rationale will be exemplified with a few individual cases below.

\section{Small molecules kinase inhibitors for the treatment of paediatric glioma}

Although this review focusses specifically on the common genetic differences between adult and paediatric patients, small molecule inhibitors of cellular pathways involved in gliomagenesis in children, in general, has been the subject of an excellent review, and includes the current challenges to small molecule therapy, and future directions in the use of this therapy. ${ }^{17}$ Similarly, the recent advances in molecular interrogation of paediatric low grade gliomas have shown a small number of recurring genetic mutations in these tumours that might be exploited therapeutically. ${ }^{18}$

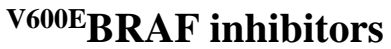

Raf kinases participate in the RAS-RAF-MEK-ERK signal transduction cascade and an exemplar inhibitor of the Raf-kinase is sorafenib. However, in the context of this review, the ${ }^{\mathrm{V} 600 \mathrm{E}} \mathrm{BRAF}$ mutation, present in approximately $20 \% \mathrm{HGA},{ }^{19}$ is an excellent case in point to highlight the way in which paediatric glioma treatment can potentially be enhanced through the design of mutation-specific small molecules (the V600E mutation results in an amino acid substitution at position 600 in BRAF, from valine (V) to glutamic acid (E)). Inhibition of BRAF signalling blocks proliferation of the cancer cells and induces apoptosis, events which have validated ${ }^{\mathrm{V} 600 \mathrm{E}} \mathrm{BRAF}$ as a therapeutic target. ${ }^{20}$ For example, recent BRAF-targeted therapies, such as vemurafenib (aka PLX4032, Figure 1), have shown great promise in 
treating V600E-dependent melanomas, such that melanoma cells without these mutations are not inhibited by vemurafenib, ${ }^{21}$ but in theory the concept could be extrapolated to other cancers with a V600E-dependence, including paediatric glioma. Evidently, the chemical differences in the structures of vemurafenib and other wild-type BRAF inhibitors is sufficient to enable vemurafenib to inhibit the mutated protein over the wild-type protein, presumably due to increased interactions between the drug and its mutated target (see examples below). Moreover, the structurally related compound, PLX4720 (Figure 1), is also a potent and selective inhibitor of ${ }^{\mathrm{V} 600 \mathrm{E}} \mathrm{BRAF}$ with an $\mathrm{IC}_{50}$ of $13 \mathrm{nM}$. This analogue has also been shown to be equally potent to c-Raf-1 (Y340D and Y341D mutations); it possesses 10-fold selectivity for the ${ }^{\mathrm{V} 600 \mathrm{E}} \mathrm{BRAF}$ mutation over wild-type B-Raf. ${ }^{22}$ Structurally vemurafenib and PLX4720 are very similar, the only difference being the ring substituent at the 5-position of the 7-azaindole ring, therefore it is not too surprising that they are both capable of inhibiting the mutant protein in a similar manner.

In addition, PLX4720 has been shown to significantly increase the survival of mice transplanted with human astrocytoma cells, ${ }^{19}$ and it has also been demonstrated that using PLX4720 in combination with PD0332991 (a CDK inhibitor) further extends survival relative to either monotherapy alone.

Based on the clear importance of developing new ${ }^{\mathrm{V} 600 \mathrm{E}} \mathrm{BRAF}$ inhibitors with increased potency and pharmacokinetic profiles, researchers at The Institute of Cancer Research, UK, developed a series of novel and potent BRAF inhibitors with nanomolar activity by way of judicious alterations to the central aromatic ring (Figure 2, 1). ${ }^{23}$ In their work, they proposed an analogous binding mode of their compounds to ${ }^{\mathrm{V} 600 \mathrm{E}} \mathrm{BRAF}$ based on the crystal structure of wild-type BRAF with sorafenib, and in doing so, they were able to exploit a lipophilic 
pocket in the binding site which would be available to accommodate the newly introduced ring substituents. All but two of the compounds prepared possessed activity that inhibited the oncogenic BRAF mutation, and in particular compounds 2 and $\mathbf{3}$ possessed $\mathrm{IC}_{50}$ values of $1 \mathrm{nM}$, results which clearly demonstrate the importance of these ring modifications in being able to inhibit ${ }^{\mathrm{V} 600 \mathrm{E}} \mathrm{BRAF}$ selectively. Importantly, several of the compounds studied achieved over 10-fold increase in activity compared to sorafenib against BRAF, results that were achieved through elaboration of the central phenyl (see $\mathbf{1}$ ) ring to capitalise on the changes to the structure and conformation of the mutant kinase.

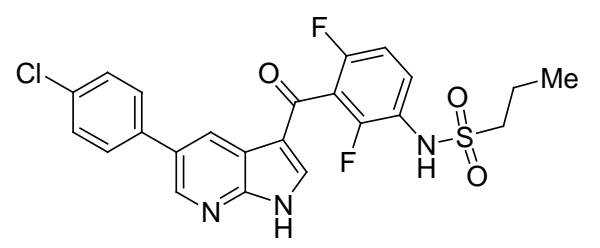

vemurafenib

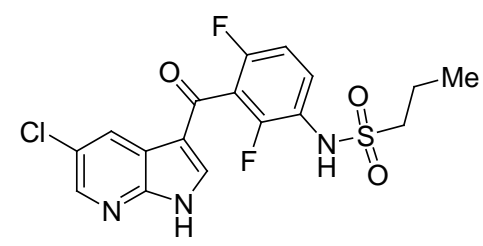

PLX4720

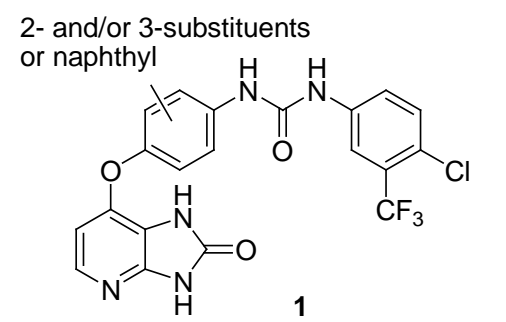<smiles>[Y][Y4]([H])([H])Cl</smiles><smiles>O=S(=O)(Nc1cc(Sc2ncn[nH]2)c(O)c2ccccc12)c1ccc(-c2ccccc2)cc1</smiles><smiles>Cc1ccc(NC(=O)c2cccc(C(C)(C)C#N)c2)cc1C(=O)Nc1cnc(C)c(Cl)c1</smiles><smiles>Cc1ccc(NC(=O)c2cccc(C(C)(C)C)c2)cc1Nc1ccc2ncn(C)c(=O)c2c1</smiles><smiles></smiles>

7<smiles>CC(=O)NC(C)c1ccc(Nc2ncc3cc(-c4ccncc4)ccc3n2)cc1</smiles>

8

${ }^{{ }^{6} 600 E_{B R A F}}{ }_{\text {IC }}{ }_{50}=77 n M$

Figure $1{ }^{\mathrm{V} 600 \mathrm{E}}$ BRAF inhibitors. 
Later, in 2012, researchers at the Wistar Institute in the U.S. identified a novel family of ${ }^{\mathrm{V} 600 \mathrm{E}}$ BRAF inhibitors based on a naphthol scaffold (Figure 3) using an ELISA-based highthroughput screening assay. These compounds possess IC 50 values in the $80-200 \mathrm{nM}$ range and are selective over wild-type BRAF. ${ }^{24}$ X-ray crystallographic studies on one of the compounds revealed that they appear to bind to the ATP binding cleft of the kinase in its active conformation, following which, a structure-activity programme was initiated on a family of naphthol compounds, resulting in compound 4 being the best candidate, which not only had significant selectivity for ${ }^{\mathrm{V} 600 \mathrm{E}}$ BRAF over wild-type BRAF, but also had selectivity over other kinases too. Again, ring-substituents appeared to be important for selective ${ }^{\mathrm{V} 600 \mathrm{E}} \mathrm{BRAF}$ inhibition, and the authors were able to identify what they believed to be a BRAF/ ${ }^{\mathrm{V} 600 \mathrm{E}} \mathrm{BRAF}$ specificity pocket into which these ring-substituents were positioned. This information could give rise to further rounds of compound development to provide extremely selective ${ }^{\mathrm{V} 600 \mathrm{E}}$ BRAF inhibitors. ${ }^{24}$

To further support this progression, researchers from AstraZeneca and Proteros Biostructures recently discovered a novel series of potent ${ }^{\mathrm{V} 600 \mathrm{E}} \mathrm{BRAF}$ selective aminoquinazoline kinase inhibitors which also demonstrated selective antiproliferative activity in mutant ${ }^{\mathrm{V} 600 \mathrm{E}} \mathrm{BRAF}$ cell lines and selectivity over other kinases (Figure 4$).{ }^{25}$ Data which supported and augmented other results from ${ }^{\mathrm{V} 600 \mathrm{E}}$ BRAF selective compounds in their collections. The development of a third series of selective ${ }^{\mathrm{V} 600 \mathrm{E}} \mathrm{BRAF}$ inhibitors at AstraZeneca serves as an additional tool to further interrogate the apparent importance of ${ }^{\mathrm{V} 600 \mathrm{E}} \mathrm{BRAF}$ within the MAPK pathway. 
Despite the knowledge that their previous compounds $\mathbf{5}$ and $\mathbf{6}$ bind to the kinase in a DFGout conformation (see Box), ${ }^{26}$ crystal structure analysis of 7 bound to a ${ }^{\mathrm{V} 600 \mathrm{E}} \mathrm{BRAF}$ model protein (EphB4: which has high binding site homology to ${ }^{\mathrm{V} 600 \mathrm{E}} \mathrm{BRAF}$ ) confirmed that 7 binds in a DFG-in conformation (see Box). ${ }^{26}$ Using this information, structural exploration began by making modifications to the A- and C-rings (Figure 4), leaving the B-ring untouched since

Box: Catalytically active kinase conformations are highly conserved due to the evolutionary pressure of maintaining function. However, inactive kinase conformations lack the same pressure and are therefore more varied across the kinase family. One specific conformation can be characterised by the unique orientation of the highly conserved Asp-Phe-Gly (DFG) motif on the kinase's activation loop. In the active conformation (DFG-in), the aspartate side-chain of the DFG motif faces into the active site to facilitate catalysis. Additionally, its neighbouring phenylalanine residue occupies a hydrophobic pocket adjacent to the ATP-binding site. In contrast, the activation loop of the observed inactive form (DFG-out) undergoes a significant translocation that moves the catalytic aspartate out of the active site and the phenylalanine away from the hydrophobic pocket. it appeared responsible for making the key interactions with the hinge region of the protein, in order to optimise potency, physical properties and in vivo rat

pharmacokinetics. From this effort lead compound $\mathbf{8}$ was identified which exhibits in vivo tumour growth inhibition in an A375 xenograft model. Interestingly, as has been observed by others, the ${ }^{\mathrm{V} 600 \mathrm{E}} \mathrm{BRAF}$ selectivity profile of these compounds resulted in the elevation of pERK. ${ }^{25}$

In addition to the experimental compounds described above, there have also been clinical trial studies performed on a relatively large number of ${ }^{\mathrm{V} 600 \mathrm{E}} \mathrm{BRAF}$ mutant kinases, and many of the compounds are now commercially available for research purposes; the subject of 'B-Raf inhibitors: from bench to bedside' has also recently been reviewed. ${ }^{27}$ 


\section{KIAA1549-BRAF}

In both normal and tumour cells which express wild-type BRAF, ${ }^{\mathrm{V} 600 \mathrm{E}} \mathrm{BRAF}$ inhibitors induce paradoxical activation of MAPK signalling which is mediated through heterodimerisation with CRAF. ${ }^{28}$ Similarly, cells expressing the KIAA1549-BRAF fusion protein demonstrate resistance and enhanced phosphorylation of MEK and ERK to first generation ${ }^{\mathrm{V} 600 \mathrm{E}}$ BRAF inhibitors, including PLX4720 (Figure 1). ${ }^{21}$ This fusion protein lacks the regulatory RAS-binding N-terminus domain and is constitutively activated through homodimerisation. The KIAA1549-BRAF fusion however is responsive to a secondgeneration selective BRAF inhibitor (PLX PB-3 - structure unavailable) that, unlike vemurafenib, does not induce activation of wild-type BRAF.

This additional complication therefore highlights the difficulties of using a 'one-method fits all' approach for the development of compounds to treat paediatric glioma. It is becoming clear that targeted treatment regimens for BRAF-altered paediatric astrocytomas are necessary, and that therapies must be tailored to the specific mutation and distinct mechanisms of action of the mutant kinase for each patient. Such a scenario may well require radical advances in the way in which patients are assessed prior to treatment.

\section{Other kinases}

With this approach in mind, arguably other kinases relevant to tumour progression could be treated in the same manner, i.e. exploiting any identified mutations to design selective treatments. For example, it is known that PDGFR- $\alpha$ activates the RAS-RAF-MEK-ERK pathway, whereby it is amplified in $30 \%$ of paediatric glioma, and of those $14 \%$ have mutations. ${ }^{29}$ Similarly, FGFR1 is a potential new target which has mutations in subsets of paediatric astrocytoma and glioblastoma multiforme. ${ }^{30}$ Drug targeting of FGFR fusions has 
shown promising results and should soon be translating into clinical trials, and further understanding of the diverse mechanisms of FGFR ought to shed light on the impact of FGFR-derived therapy in the future. ${ }^{30}$

The PI3K/AKT/mTOR pathway is an intracellular signalling pathway important in apoptosis, and consequently cancer, and the pathway is upregulated in both low and high grade astrocytoma. Within this pathway, a number of targets have been identified which are currently under examination.

\section{IGF1R}

The insulin-like growth factor 1 receptor (IGF1R) has been identified as a potential therapeutic target in paediatric glioblastoma due to gene amplification and high levels of IGF2 expression in some tumour samples. ${ }^{31}$ In 2011, Bielen and co-workers evaluated the therapeutic potential of targeting this receptor by carrying out in vitro and in vivo preclinical studies using the specific IGF1R inhibitor NVP-AEW541 (Figure 5). GI50 values of 5-6 $\mu \mathrm{M}$, and concurrent inhibition of receptor phosphorylation were observed along with hallmarks of PI3K inhibition after treatment with the compound. ${ }^{31}$ Furthermore, their studies show that cotreatment of cells with NVP-AEW541 and imatinib resulted in a highly synergistic interaction in vitro compared with either agent alone. Identifying other kinases that may also be actively involved in paediatric glioblastoma cell lines will also help to design appropriate combination strategies to enhance IGFR1 inhibitor treatment. 


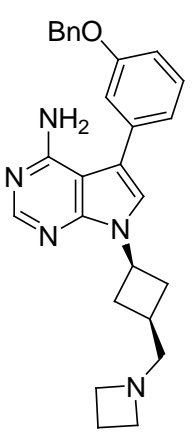

NVP-AEW541

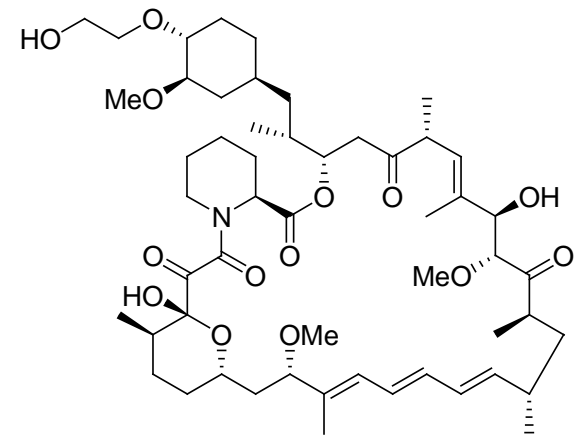

everolimus<smiles>COCCOCCOC</smiles>

10

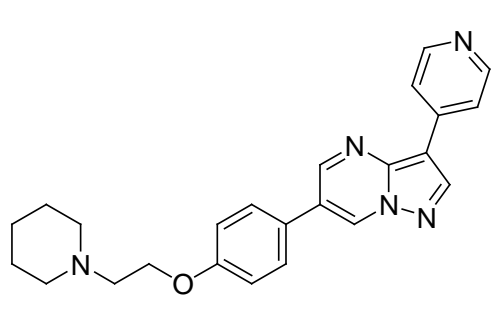

dorsomorphin<smiles>CCCCCCCCCCCCCCCCCC(C)(C)C</smiles><smiles>COc1cc(-c2cc(-c3cccc(O)c3)cnc2N)cc(OC)c1OC</smiles>

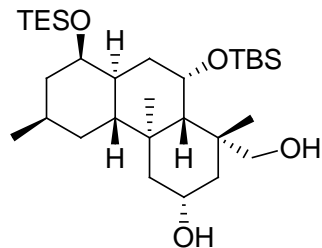

SPI-001<smiles>COCCOCc1cc(F)ccc1-c1nc(-c2ccc3[nH]c(=O)[nH]c3c2)cs1</smiles>

11

Figure 2 Other kinase inhibitors and the phosphatase inhibitors SPI-001 and 11.

\section{mTOR}

The mechanistic target of rapamycin (mTOR) is a serine threonine protein kinase that regulates cell growth, cell proliferation, cell motility, cell survival, protein synthesis, and transcription, and effective pharmacologic inhibitors such as rapamycin (and analogues, socalled rapalogues) have been identified. One such analogue is everolimus (Figure 5) which is licensed in children from the age of 3 years for the treatment of subependymal giant cell astrocytoma associated with tuberous sclerosis complex who require therapeutic intervention but are not amenable to surgery. 
Hütt-Cabezas has recently reviewed the topic in a paper titled 'Activation of mTORC1/mTORC2 signaling in pediatric low-grade glioma and pilocytic astrocytoma reveals mTOR as a therapeutic target', ${ }^{32}$ and the importance of this pathway is also important in children with hematologic malignancies too. ${ }^{33}$

Based on the pressing need to develop new inhibitors for mTOR, a number of researchers are currently identifying and developing novel small molecules against this, and related, targets. For example, Lin and co-workers have developed a series of novel 7-amino-5-methyl-1,6naphthyridin-2(1H)-one derivatives (9, Figure 6) as potent (nanomolar) PI3K/mTOR dual inhibitors. $^{34}$

Basing their compounds on a 4-methylpyridopyrimidinone scaffold the researchers hoped to identify a new inhibitor template that could address some of the issues with current treatments. Upon examining the co-crystal structure of 4-methylpyridopyrimidinone and PI3K they noticed that there was no direct interaction between the nitrogen atom at $\mathrm{N}-1$ and PI3K, which immediately identified a good starting point to diversify the scaffold (see $\mathrm{R}^{1}$ on 9). ${ }^{34}$ This approach aligns well with the concept of designing new inhibitors for mutant targets and harnessing an under-exploited interaction to yield potent inhibitors. Their series of compounds, which turned out to be dual PI3K/mTOR inhibitors, had potent nanomolar IC $_{50}$ S which were comparable to a related compound in Phase two clinical trials, as well as acceptable cellular activities and pharmacokinetic profiles. Importantly, the authors suggest that identifying this series gives rise to a broader chemical space becoming available and thus improved opportunities for developing anti-tumour agents and advancing them into clinical trials. 
Conversely, Lee and co-workers explored a series of $N$-7-methyl-imidazolopyrimidines based on the hypothesis that the $N$-7-methyl substituent on the imidazolopyrimidine motif would impart selectivity for mTOR over the related PI3K alpha and delta kinases. ${ }^{35}$ The corresponding $N$-Me substituted pyrrolo[3,2- $d$ ]pyrimidines and pyrazolo[4,3- $d$ ]pyrimidines (10, Figure 6) also show potent mTOR inhibition with selectivity toward both PI3 alpha and delta kinases. The most potent compound (10) possesses a $K_{i}$ of $2 \mathrm{nM}$ against mTOR inhibition, and significant selectivity (>2900x) over PI3 kinases, as well as possessing excellent potency in cell-based assays. ${ }^{35}$ The syntheses of PI3K/Akt/mTOR signaling pathway inhibitors has also recently been reviewed. ${ }^{36}$

\section{Other targets}

Diffuse gliomas in adults generally arise in cerebral white matter, whereas paediatric cases often arise in deep cerebral structures, most commonly the brainstem. In contrast to adult tumours, childhood tumours commonly have mutations in histone H3 genes (H3F3A and HIST1H3B), usually encoding a p.Lys27Met substitution. ${ }^{37}$ Recent studies have also recognised recurrent mutations of the $A C V R 1$ gene in these tumours, thereby highlighting another important difference between gliomas affecting children and adults and a potential target for developing much needed therapies. ${ }^{15}$ Taylor et al. have identified mutations in the ACVR1 gene, which encodes a type I activin receptor serine/threonine kinase (ALK), in 21\% of DIPG samples. ${ }^{15}$ Overall, the researchers identified 11 of 52 DIPG samples (21\%) possessing mutations in ACVR1 which appear to be highly specific to DIPG.

Treatment of four case-derived DIPG primary cultures with the selective ALK2 inhibitor LDN-193189 (Figure 7) resulted in noteworthy inhibition of cell viability in all cells, with $\mathrm{GI}_{50}$ values ranging from $0.86-2.1 \mu \mathrm{M}$. This value was approximately tenfold lower than with 
the structurally related parent compound dorsomorphin (Figure 7), with a trend toward increased sensitivity in the mutant cultures. Transfection of affected cells with wild-type ACVR1 conferred increased signalling through phosphorylated SMAD1/5/8, particularly for the Arg206His mutant and, to a lesser extent, for the Gly328Glu mutant.

Dorsomorphin analogues remain the only ALK2 inhibitors reported to date. ${ }^{38}$ Therefore, in attempts to address this shortfall, Sanvitale et al. screened a library of 250 recombinant human kinases, and identified a highly selective 2-aminopyridine-based inhibitor (K02288, Figure 7) with in vitro activity against ALK2 at low nanomolar concentrations, values which they found to be similar to the benchmark compound LDN-193189. As would be hoped when developing new inhibitors, comparison of the crystal structures of ALK2 with K02288 and LDN-193189 revealed additional contacts between the protein and K02288, giving rise to improved binding, but also identified an exposed phenol group (see text in Figure 7) for further optimisation. For the authors, the discovery of this new chemical series provided a pharmacological tool to investigate bone morphogenetic protein (BMP), a protein of importance to the congenital childhood developmental disorder fibrodysplasia ossificans progressiva (FOP) ${ }^{38}$ However, this is a disease which has mutations in the germ line of individuals which are identical to mutations found in cancer and so these new lead compounds may find success in the treatment of childhood DIPG too.

In their work identifying all the protein-coding genes in certain tumours, Zhang et al. performed exomic sequencing of 14 brainstem gliomas (BSGs, of which 75\% are diagnosed in children and young adults) and 12 thalamic gliomas. Their results identified tumour-specific mutations in PPM1D in 37.5\% of the BSGs that harboured hallmark H3F3A 
mutations encoding p.Lys27Met substitutions, results which define PPM1D as a potential therapeutic target in brainstem gliomas. ${ }^{39}$

Protein phosphatase 1D is an enzyme that in humans is encoded by the PPM1D gene. The protein is a Ser/Thr protein phosphatase which is capable of negatively regulating the activity of p38 MAP kinase (MAPK/p38) whereby it reduces the phosphorylation of p53, and in turn suppresses p53-mediated transcription and apoptosis. Based on such a mechanism, the phosphatase activity of PPM1D is essential for its oncogenic role, and thus PPM1D inhibitors should be viable anti-cancer agents. Based on this proposal, Yagi and co-workers have developed a small molecule inhibitor of PPM1D based on a perhydrophenanthrene scaffold (Figure 8) which was identified by screening their chemical library for PPM1D phosphatase activity. ${ }^{40}$ The best compound, SPI-001, exhibits structural diversity from other known PPM1D inhibitors and represents a new scaffold for this target. The compound demonstrates very strong inhibition against PPM1D with a sub-micromolar IC50 value $(0.48 \pm 0.04 \mu \mathrm{M})$. SPI-001 suppresses the cell proliferation of MCF7 cells by inducing apoptosis, whilst structure-activity relationships indicated that the two hydrophobic moieties (TES and TBS in SPI-001) are important for inhibitory activity.

Furthermore, in 2014, The Institute of Cancer Research, UK, identified 2,4-bisarylthiazoles (e.g. 11, Figure 8) as being able to cause highly selective apoptosis in PPM1D amplified celllines. ${ }^{41}$ Having optimized the 2,4-bisarylthiazole structure for selective growth-inhibition of the PPM1D amplified cell-line SMOV2 versus the non-amplified cell-line TOV21G, the group decided to assess how general this PPM1D amplification phenotype was. In doing so, compound 11 was examined in a 5-day sulforhodamine B (SRB) growth inhibition assay against various PPM1D amplified and non-amplified cell-lines. None of the non-amplified 
cell-lines were sensitive to 11, whilst all three PPM1D amplified cell-lines displayed high sensitivity to the same compound, leading to potent low nanomolar growth-inhibition. Their class of compound displays clear structure-activity relationships, with particular importance for the cellular activity associated with the 4-fluorophenyl substituent. ${ }^{41}$

\section{WHERE NEXT?}

Due to the increasing number of genetic variations that continue to be identified between adult and paediatric glioma, it is becoming clear that tailored therapies to an individual child's genetic requirements may be desirable. Identifying suitable biomarkers to these variations would therefore be of utmost importance in order to maximise the efficacy of treatment. That said, it can take up to 15 years to get a drug from the experimental stages to the clinic, therefore, identifying mutant targets and developing inhibitors of them as soon as possible is crucial so that an arsenal of compounds can be available for all contingencies. However, monotherapy alone using selective kinase (or other target) inhibitors, is unlikely to prove completely effective due to intrinsic or acquired resistance problems seen with other anticancer drugs. Nevertheless, overcoming such limitations could possibly be mitigated using combination treatments which target alternative oncogenic pathways, using either current drug combinations or new drugs with existing ones.

\section{ACKNOWLEDGEMENTS}

This work was part-funded by Brain Tumour North West and the authors would like to thank them. 


\section{REFERENCES}

1. Louis DN, Ohgaki H, Wiestler OD, et al.: The 2007 who classification of tumours of the central nervous system, Acta Neuropathol, 114: 97-109, 2007.

2. Walker DA, Liu J, Kieran M, et al.: A multi-disciplinary consensus statement concerning surgical approaches to low-grade, high-grade astrocytomas and diffuse intrinsic pontine gliomas in childhood (CPN Paris 2011) using the delphi method, Neuro-Oncology, 15: 462-468, 2013.

3. Benesch M, Lackner H, Sovinz P, et al.: Late sequela after treatment of childhood lowgrade gliomas: A retrospective analysis of 69 long-term survivors treated between 1983 and 2003, J Neurooncol, 78: 199-205, 2006.

4. Wolff JEA, Driever PH, Erdlenbruch B, et al.: Intensive chemotherapy improves survival in pediatric high-grade glioma after gross total resection: Results of the hitgbm-c protocol, Cancer, 116: 705-712, 2010.

5. Ater JL, Zhou T, Holmes E, et al.: Randomized study of two chemotherapy regimens for treatment of low-grade glioma in young children: A report from the children's oncology group, J Clin Oncol, 30: 2641-2647, 2012.

6. Warren KE, Gururangan S, Geyer JR, et al.: A phase II study of $\mathrm{O}^{6}$-benzylguanine and temozolomide in pediatric patients with recurrent or progressive high-grade gliomas and brainstem gliomas: A pediatric brain tumor consortium study, J Neurooncol, 106: 643-649, 2012.

7. Zhang J, Wu G, Miller CP, et al.: Whole-genome sequencing identifies genetic alterations in pediatric low-grade gliomas, Nat Genet, 45: 602-612, 2013.

8. Schiffman JD, Hodgson JG, VandenBerg SR, et al.: Oncogenic BRAF mutation with CDKN2A inactivation is characteristic of a subset of pediatric malignant astrocytomas, Cancer Res, 70: 512-519, 2010. 
9. Jones DTW, Hutter B, Jaeger N, et al.: Recurrent somatic alterations of FGFR1 and NTRK2 in pilocytic astrocytoma, Nat Genet, 45: 927-932, 2013.

10. Fontebasso AM, Papillon-Cavanagh S, Schwartzentruber J, et al.: Recurrent somatic mutations in ACVR1 in pediatric midline high-grade astrocytoma, Nat Genet, 46: 462466, 2014.

11. Tatevossian RG, Tang B, Dalton J, et al.: MYB upregulation and genetic aberrations in a subset of pediatric low-grade gliomas. Acta Neuropathol, 120: 731-743, 2010.

12. Ramkissoon LA, Horowitz PM, Craig JM, et al.: Genomic analysis of diffuse pediatric low-grade gliomas identifies recurrent oncogenic truncating rearrangements in the transcription factor MYBL1, Proc Natl Acad Sci USA, 110: 8188-8193, 2013.

13. Wu G, Diaz AK, Paugh BS, et al.: The genomic landscape of diffuse intrinsic pontine glioma and pediatric non-brainstem high-grade glioma. Nat Genet, 46: 444-450, 2014.

14. Buczkowicz P, Hoeman C, Rakopoulos P, et al.: Genomic analysis of diffuse intrinsic pontine gliomas identifies three molecular subgroups and recurrent activating ACVR1 mutations. Nat Genet, 46: 451-456, 2014.

15. Taylor KR, Mackay A, Truffaux N, et al.: Recurrent activating ACVR1 mutations in diffuse intrinsic pontine glioma, Nat Genet, 46: 457-461, 2014.

16. Zhang J, Yang PL, Gray NS: Targeting cancer with small molecule kinase inhibitors, Nat. Rev. Cancer, 9: 28-39, 2009.

17. Herrington B, Kieran MW: Small molecule inhibitors in children with malignant gliomas, Pediatr Blood Cancer, 53: 312-317, 2009.

18. Raabe E, Kieran MW, Cohen KJ: New strategies in pediatric gliomas: Molecular advances in pediatric low-grade gliomas as a model, Clin Can Res, 19: 4553-4558, 2013. 
19. Huillard E, Hashizume R, Phillips JJ, et al.: Cooperative interactions of BRAF(V600E) kinase and CDKN2A locus deficiency in pediatric malignant astrocytoma as a basis for rational therapy. Proc Natl Acad Sci USA, 109: 8710-8715, 2012.

20. Karasarides M, Chiloeches A, Hayward R, et al.: BRAF is a therapeutic target in melanoma, Oncogene, 23: 6292-6298, 2004.

21. Sievert AJ, Lang S-S, Boucher KL, et al.: Paradoxical activation and RAF inhibitor resistance of BRAF protein kinase fusions characterizing pediatric astrocytomas, Proc Natl Acad Sci USA, 110: 5957-5962, 2013.

22. Tsai J, Lee JT, Wang W, et al.: Discovery of a selective inhibitor of oncogenic B-RAF kinase with potent antimelanoma activity, Proc Natl Acad Sci USA, 105: 3041-3046, 2008.

23. Menard D, Niculescu-Duvaz I, Dijkstra HP, et al.: Novel potent BRAF inhibitors: Toward $1 \mathrm{~nm}$ compounds through optimization of the central phenyl ring, J Med Chem, 52: 3881-3891, 2009.

24. Qjn J, Xie P, Ventocilla C, et al.: Identification of a novel family of BRAF(V600E) inhibitors, J Med Chem, 55: 5220-5230, 2012.

25. Vasbinder MM, Aquila B, Augustin M, et al.: Discovery and optimization of a novel series of potent mutant BRAF(V600E) selective kinase inhibitors, J Med Chem, 56: 1996-2015, 2013.

26. Hari SB, Merritt EA, Maly DJ: Sequence determinants of a specific inactive protein kinase conformation, Chem Biol, 20: 806-815, 2013.

27. Huang T, Karsy M, Zhuge J, Zhong M, Liu D: BRAF and the inhibitors: From bench to bedside, J Hematol Oncol, 6: 30, 2013. 
28. Joseph EW, Pratilas CA, Poulikakos PI, et al.: The RAF inhibitor PLX4032 inhibits ERK signaling and tumor cell proliferation in a V600E BRAF-selective manner, Proc Natl Acad Sci USA, 107: 14903-14908, 2010.

29. Paugh BS, Zhu XY, Qu CX, et al.: Novel oncogenic PDGFRA mutations in pediatric high-grade gliomas, Cancer Res, 73: 6219-6229, 2013.

30. Parker BC, Engels M, Annala M, Zhang W: Emergence of FGFR family gene fusions as therapeutic targets in a wide spectrum of solid tumours, J Pathol, 232: 4-15, 2014.

31. Bielen A, Perryman L, Box GM, et al.: Enhanced efficacy of IGF1R inhibition in pediatric glioblastoma by combinatorial targeting of PDGFR $\alpha / \beta$, Mol Cancer Ther, 10 : 1407-1418, 2011.

32. Huett-Cabezas M, Karajannis MA, Zagzag D, et al.: Activation of MTORC1/MTORC2 signaling in pediatric low-grade glioma and pilocytic astrocytoma reveals MTOR as a therapeutic target, Neuro-Oncology, 15: 1604-1614, 2013.

33. Barrett D, Brown VI, Grupp SA, Teachey DT: Targeting the PI3K/AKT/MTOR signaling axis in children with hematologic malignancies, Pediatr Drugs, 14: 299-316, 2012.

34. Lin S, Han F, Liu P, et al.: Identification of novel 7-amino-5-methyl-1,6-naphthyridin2(1h)-one derivatives as potent PI3K/MTOR dual inhibitors, Bioorg Med Chem Lett, 24: 790-793, 2014.

35. Lee W, Ortwine DF, Bergeron P, et al.: A hit to lead discovery of novel N-methylated imidazolo-, pyrrolo-, and pyrazolo-pyrimidines as potent and selective MTOR inhibitors, Bioorg Med Chem Lett, 23: 5097-5104, 2013.

36. Welker ME, Kulik G: Recent syntheses of PI3K/AKT/MTOR signaling pathway inhibitors, Bioorg Med Chem, 21: 4063-4091, 2013. 
37. Zadeh G, Aldape K: ACVR1 mutations and the genomic landscape of pediatric diffuse glioma, Nat Genet, 46: 421-422, 2014.

38. Sanvitale CE, Kerr G, Chaikuad A, et al.: A new class of small molecule inhibitor of BMP signaling, Plos One, 8: 4, e62721- e62721, 2013.

39. Zhang L, Chen LH, Wan H, et al.: Exome sequencing identifies somatic gain-offunction PPM1D mutations in brainstem gliomas, Nat Genet, 46: 726-730, 2014.

40. Yagi H, Chuman Y, Kozakai Y, et al.: A small molecule inhibitor of p53-inducible protein phosphatase PPM1D, Bioorg Med Chem Lett, 22: 729-732, 2012.

41. Cheeseman MD, Faisal A, Rayter S, et al.: Targeting the PPM1D phenotype; 2,4bisarylthiazoles cause highly selective apoptosis in PPM1D amplified cell-lines, Bioorg Med Chem Lett, 24: 3469-3474, 2014.

\section{LEGENDS}

Figure $1{ }^{\mathrm{V} 600 \mathrm{E}} \mathrm{BRAF}$ inhibitors.

Figure 2 Other kinase inhibitors and the phosphatase inhibitors SPI-001 and 11. 